\title{
Secretor status and Helicobacter pylori infection are independent risk factors for gastroduodenal disease
}

\author{
W Dickey, J S A Collins, R G P Watson, J M Sloan, K G Porter
}

\begin{abstract}
The hypothesis that non-secretors of ABO blood group antigens, a group shown to be more susceptible to certain bacterial infections, may be at greater risk of gastroduodenal disease because of increased susceptibility to Helicobacter pylori infection was investigated. Of 101 patients with symptoms of dyspepsia who were undergoing endoscopy, $32 \%$ were non-secretors (determined from Lewis blood group phenotype), $36 \%$ had endoscopically visible gastroduodenal disease (antral gastritis, gastric ulcer, erosive duodenitis, duodenal ulcer or some combination), and $58 \%$ had $H$ pylori detected in antral biopsy specimens. Non-secretors and patients with $H$ pylori infection were significantly more likely to have gastroduodenal disease $(p=0.02$ and $p=0.002$ respectively). There was, however, no significant association between secretor status and $H$ pylori infection, logistic regression analysis confirming that these were independently associated with gastroduodenal disease. Overall, the relative risk of gastroduodenal disease for non-secretors compared with secretors was 1.9 (95\% confidence intervals $1 \cdot 2,3 \cdot 2)$. Non-secretion of ABO blood group antigens is not related to $H$ pylori infection but is independently and significantly associated with endoscopic gastroduodenal disease. The mechanism of this remains to be explained. (Gut 1993; 34: 351-353)
\end{abstract}

Non-secretors of $\mathrm{ABO}$ blood group antigens into body fluids are known to be at increased risk of a variety of bacterial and fungal infections' and are also more prone to duodenal ulcer.' As Helicobacter pylori infection is strongly associated with duodenal ulcer, duodenitis, and antral gastritis, we wished to determine whether the risk of gastroduodenal disease in general was related to secretor status. We also wished to test the hypothesis that any increased risk in nonsecretors might be caused by increased susceptibility to $H$ pylori infection by studying patients with dyspeptic symptoms who had been referred for upper gastrointestinal endoscopy.

Patients and methods

We studied 106 consecutive patients referred for endoscopy as part of an investigation of dyspeptic symptoms, who had not received antibiotics, $\mathrm{H}_{2}$ receptor antagonists, or omeprazole during the previous 4 weeks and who had never taken bismuth preparations. Lewis phenotype was used to determine secretor status: blood was tested for Lewis antigens by standard tube agglutination methods with monoclonal anti-
Lewis a and anti-Lewis b antibodies. Nonsecretors express the Lewis a antigen only and secretors can express the Lewis b antigen.' At endoscopy, biopsy specimens were taken from the gastric antrum; these were tested for the presence of $H$ pylori by commercial urease (CLO) test and examined histologically using Giemsa staining.

The $\chi^{2}$ test with Yate's correction was used to analyse discontinuous variables. Logistic regression and relative risk estimation were performed using the EGRET and EpiInfo packages on an IBM/PC compatible microcomputer.

\section{Results}

Five of the 106 patients studied were both Lewis $a$ and $b$ antigen negative. As secretor status cannot be inferred from this phenotype these patients were excluded from further analysis. The remaining 101 patients had ages ranging from 19 to 70 years (mean 45 ) and 48 were men. Sixty nine $(68 \%)$ patients were secretors and 32 were non-secretors. The endoscopic diagnosis was some combination of antral gastritis, erosive duodenitis, gastric ulcer, and duodenal ulcer in $36(36 \%)$ patients: these were considered to have significant gastroduodenal disease. Nine of these had duodenal ulcer and one had gastric ulcer. In $53(52 \%)$ patients endoscopy was normal, and 12 had reflux oesophagitis. Fifty nine (58\%) patients had $H$ pylori detected in antral biopsy specimens.

Non-secretors were significantly more likely to have endoscopically visible gastroduodenal disease (17 (53\%) of 32 compared with $19(28 \%)$ of 69 secretors: $\left.\chi^{2}=5 \cdot 17, \mathrm{DF}=1, \mathrm{p}=0 \cdot 02\right)$. As expected, $H$ pylori infection was also significantly associated with gastroduodenal disease $(29(49 \%)$ of 59 compared with $7(17 \%)$ of $42 \mathrm{H}$ pylori negative patients: $\chi^{2}=9 \cdot 92, \mathrm{DF}=1, \mathrm{p}=0.002$ ).

There was no significant association between secretor status and $H$ pylori infection, however, with $H$ pylori present in $39(57 \%)$ of 69 secretors and $20(63 \%)$ of 32 non-secretors $\left(\chi^{2}=0 \cdot 12\right.$, $\mathrm{DF}=1, \mathrm{p}=0.73)$.

Among the $H$ pylori positive patients, $13(65 \%)$ of 20 non-secretors had endoscopically visible gastroduodenal disease compared with $16(41 \%)$ of 39 secretors, although this difference failed to reach statistical significance $\left(\chi^{2}=2 \cdot 16, D F=1\right.$, $\mathrm{p}=0 \cdot 14)$. In order to confirm that $H$ pylori infection and secretor status were independent risk factors for gastroduodenal disease, we performed a logistic regression analysis on the data obtained. The results confirmed that there was no significant interaction between the presence of $H$ pylori and secretor status with regard to endoscopically visible gastroduodenal pathology findings (Table I). 
TABLE I Logistic regression model testing for an interaction between secretor status and $\mathbf{H}$ pylori infection with regard to gastroduodenal disease

\begin{tabular}{lllll}
\hline & Coefficient & $S E M$ & $p$ & Odds ratio \\
\hline Univariate analysis: & & & & \\
H pylori +ve & 1.576 & 0.489 & 0.001 & 4.833 \\
$\quad$ Non-secretor & 1.093 & 0.445 & 0.014 & 2.982 \\
Multivariate analysis: & & & & \\
H pylori +ve & 1.612 & 0.506 & 0.001 & 5.014 \\
Non-secretor & 1.144 & 0.479 & 0.017 & 3.139 \\
\hline
\end{tabular}

Test for interaction: $\mathrm{X}^{2}=0 \cdot 256, \mathrm{DF}=1, \mathrm{p}=0 \cdot 61$.

TABLE II Risk of gastroduodenal disease in patients with dyspepsia referred for endoscopy by secretor and $\mathrm{H}$ pylori status relative to $\mathrm{H}$ pylori negative secretors

\begin{tabular}{lll}
\hline Patient status & $\begin{array}{l}\text { Relative } \\
\text { risk }\end{array}$ & $\begin{array}{l}95 \% \text { Confidence } \\
\text { intervals }\end{array}$ \\
\hline H pylori - ve/secretor & $1 \cdot 0$ & \\
H pylori - ve/non-secretor & $3 \cdot 3$ & $(0 \cdot 9-12 \cdot 7)$ \\
H pylori +ve/secretor & $4 \cdot 1$ & $(1 \cdot 3-12 \cdot 8)$ \\
H pylori +ve/non-secretor & 6.5 & $(2 \cdot 1-19 \cdot 9)$ \\
\hline
\end{tabular}

TABLE III Risk of gastroduodenal disease in non-secretors relative to secretors, categorised by $\mathbf{H}$ pylori status

\begin{tabular}{lll}
\hline H pylori status & Relative risk ${ }^{\star}$ & $95 \%$ Confidence intervals \\
\hline -ve & 3.3 & $(0 \cdot 9-12 \cdot 7)$ \\
+ve & 1.6 & $(1 \cdot 0-2 \cdot 6)$ \\
All patients & 1.9 & $(1 \cdot 2-3 \cdot 2)$ \\
\hline
\end{tabular}

${ }^{\star}$ Relative risk for secretors in each category $=1.0$.

The relative risks for gastroduodenal disease among patients, depending on secretor and $H$ pylori status, are shown in Tables II and III. Overall, the relative risk for non-secretors compared with secretors, irrespective of $H$ pylor status, was $1.9(95 \%$ confidence intervals 1.2 $3 \cdot 2)$.

\section{Discussion}

Non-secretion of $\mathrm{ABO}$ blood group antigens into body fluids has been shown to be significantly associated with susceptibility to rheumatic fever and rheumatic heart disease, ${ }^{3}$ recurrent urinary tract infections, ${ }^{4}$ infections caused by pneumococcus, meningococcus, and Haemophilus influenzae, ${ }^{56}$ cholera, ${ }^{7}$ and oral candida infection. ${ }^{89}$ In addition, non-secretors have a significantly increased risk of duodenal ulcer, ${ }^{2}$ and a 1965 study showed that among duodenal ulcer patients, non-secretors were more likely to undergo surgery. ${ }^{10}$ In our own group of patients, non-secretors exhibited some combination of endoscopically visible antral gastritis, gastric ulcer, duodenitis, and duodenal ulcer significantly more often than secretors. 'Although we have no figures for the prevalence of nonsecretors in Northern Ireland, that of patients without endoscopic gastroduodenal disease in this study (23\%: 15 of 65 ) is comparable with figures reported elsewhere in the British Isles:' in contrast, the prevalence of non-secretors in patients with endoscopic abnormalities was $47 \%$ (17 of 36).

Most previous studies were undertaken before the widespread use of endoscopy and therefore included only ulcer disease diagnosed radiologically or surgically.

The hypothesis that the increased prevalence of gastroduodenal disease in non-secretors is associated with and results from an increased susceptibility to $H$ pylori infection is attractive, and some studies have addressed this possibility. Hook-Nikanne et $a l^{11}$ studied $H$ pylori serology and secretor status in 271 blood donors and found no association: endoscopy was not performed. Similarly, Chesner et al ${ }^{12}$ determined secretor and $H$ pylori status in 185 patients with dyspepsia but not peptic ulcer and showed no significant association: the relative risk of $H$ pylori infection in non-secretors compared with secretors was 0.95 (95\% confidence intervals $0 \cdot 49,1 \cdot 84)$. We are aware of only one other study that has attempted to correlate the endoscopic appearances with secretor status. Mentis et al, ${ }^{13}$ like us, failed to show an association between secretor status and $H$ pylori infection among 454 patients studied endoscopically, but in addition they found no association between secretor status and either gastric or duodenal ulcer. They did not state, however, how many of their patients without ulcer had gastritis or duodenitis, and may therefore have compared a heterogenous control group, some of whom had abnormalities that we have shown to be linked to secretor status, with the ulcer patients.

We have shown that non-secretion of blood group antigens is a significant risk factor (relative risk 1.9 for all patients) for gastroduodenal disease, including antral gastritis and duodenitis without ulcer, in patients with dyspeptic symptoms studied endoscopically. Non-secretion is not associated with the presence of $H$ pylori in antral biopsy specimens and does not therefore seem to be associated with increased susceptibility to infection with this organism, although secretor status may determine the host response to colonisation. The mechanism remains to be determined: some reports of reduced levels of serum and salivary IgA in non-secretors, ${ }^{14}$ suggesting that mucosal protection may be abnormal, have not been substantiated by other workers. ${ }^{15}$

We thank Dr C Patterson, Department of Epidemiology and Public Health, Queen's University of Belfast, for statistical advice and the Northern Ireland Blood Transfusion Service who tested blood samples for Lewis antigens.

Presented at British Society of Gastroenterology Autumn Meeting, 9 September 1992

1 Blackwell CC. Genetic susceptibility to infectious agents. Pro $R$ Coll Phys Edinb 1989; 19: 129-38.

2 Clarke CA, Wyn Edwards J, Haddock DRW, Howel-Evans AW, McConnell RB, Sheppard PM. ABO blood groups and AW, Mccorner character in duodenal ulcer. BMF 1956;2:725-31.

3 Haver groups, and secretor status. Am F Hum Genet 1969; 21: 360 75 .

4 Kinane DF, Blackwell CC, Brettle RP, Weir DM, Winstanley FP, Elton RA. ABO blood group, secretor state, and FP, Elton RA. ABO blood group, secretor state, and susceptibility to recur

5 Blackwell CC, Jonsdottir K, Hanson $M$, Todd WTA Chaudhuri AKR, Mathew B, et al. Non-secretion of ABO blood group antigens predisposing to infection by Neisseria meningitidis and Streptococcus pneumoniae. Lancet 1986 ii: $284-5$

6 Blackwell CC, Jonsdottir K, Hanson MF, Weir DM. Nonsecretion of $\mathrm{ABO}$ blood group antigens predisposing to infection by Haemophilus influenzae. Lancet 1986; ii: 687 .

7 Chaudhuri A, Das Adhikary CR. Possible role of bloo secretory substances in the aetiology of cholera. Trans $R$ So Trop Med Hyg 1978; 72: 664-5.

8 Thom SM, Blackwell CC, MacCallum CJ, Weir DM, Brettle RP, Kinane DF, et al. Non-secretion of blood group antigens and susceptibility to infections by Candida species. antigens and susceptibility to infections by

9 Aly FZ, Blackwell CC, MacKenzie DAC, Weir DM, Elton 
RA, Cumming CG, et al. Chronic atrophic oral candidiasis among patients with diabetes mellitus - role of secreto status. Epidemiol Infect 1991; 106: 355-63.

10 Langman MJS, Doll R. ABO blood group and secretor status in relation to clinical characteristics of peptic status in relation to

11 Hook-Nikeanne J, Sistonen P, Kosunen TU. Effect of ABO blood group and secretor status on the frequency of Helicobacter pylori antibodies. Scand $\mathcal{F}$ Gastroenterol 1990; 25: 815-8

12 Chesner IM, Nicholson G, Ala F, Elliott TSJ, Langman MJS Predisposition to gastric mucosal infection by Helicobacter pylori: an investigation of any association with $\mathrm{ABO}$ or
Lewis blood group and secretor status. Eur $\mathcal{f}$ Gastroenterol Hepatol 1992; 4: 377-9.

13 Mentis A, Blackwell CC, Weir DM, Spiladis C, Dailianas A, Skandalis N. ABO blood group, secretor status and detection of Helicobacter pylori among patients with gastric or duodenal ulcers. Epidemiol Infect 1991; 106: 221-9.

14 Waissbluth JG, Langman MJS. ABO blood groups, secretor status, salivary protein, and serum and salivary immunostatus, salivary protein, and serum and sali

15 Blackwell CC, Jonsdottir K, Weir DM, Hanson MF, Cartwright KAV, Stewart J, et al. Blood groups, secretor status and susceptibility to bacterial meningitis. FEMS Microbiol Immunol 1989; 47: 351-6. 